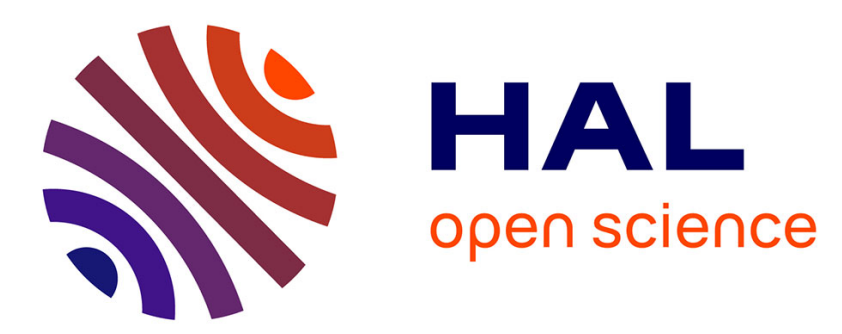

\title{
Peculiar effective elastic anisotropy of nanometric multilayers studied by surface Brillouin scattering
}

D Faurie, P Djemia, O Castelnau, R Brenner, Eric Le Bourhis, L Belliard, P. Goudeau, P.-O Renault

\section{- To cite this version:}

D Faurie, P Djemia, O Castelnau, R Brenner, Eric Le Bourhis, et al.. Peculiar effective elastic anisotropy of nanometric multilayers studied by surface Brillouin scattering. Superlattices and Microstructures, 2015, 88, pp.551-560. 10.1016/j.spmi.2015.10.015 . hal-01326100

\section{HAL Id: hal-01326100 \\ https://hal.science/hal-01326100}

Submitted on 11 Jul 2017

HAL is a multi-disciplinary open access archive for the deposit and dissemination of scientific research documents, whether they are published or not. The documents may come from teaching and research institutions in France or abroad, or from public or private research centers.
L'archive ouverte pluridisciplinaire HAL, est destinée au dépôt et à la diffusion de documents scientifiques de niveau recherche, publiés ou non, émanant des établissements d'enseignement et de recherche français ou étrangers, des laboratoires publics ou privés. 


\title{
Peculiar effective elastic anisotropy of nanometric multilayers studied by surface Brillouin scattering
}

\author{
D. Faurie ${ }^{\text {a, * }}$, P. Djemia ${ }^{\text {a }}$, O. Castelnau ${ }^{\text {b }}$, R. Brenner ${ }^{\text {c }}$, L. Belliard ${ }^{\mathrm{d}}$, \\ E. Le Bourhis ${ }^{\text {e, }}$ Ph Goudeau ${ }^{\text {e }}$, P.-O. Renault ${ }^{\mathrm{e}}$ \\ a LSPM-CNRS, Université Paris XIII, Sorbonne Paris Cité, 93430 Villetaneuse, France \\ b PIMM, CNRS-ENSAM Paris, F-75015 Paris, France \\ c Institut Jean Le Rond D'Alembert, CNRS-Université Pierre et Marie Curie, F-75006 Paris, France \\ d INSP, CNRS-Université Pierre et Marie Curie, F-75006 Paris, France \\ e Institut Pprime, CNRS, Université de Poitiers, UPR 3346, Département de Physique et Mécanique des Matériaux, BP 30179,86962 \\ Futuroscope, France
}

Keywords:

Elastic anisotropy

Multilayers

Homogenization

\begin{abstract}
A B S T R A C T
We show in this paper by using a two-scale transition model that the elastic anisotropy of a thin film specimen can be tuned by appropriate stacking design. The anisotropic behaviour is illustrated for two monophase thin films, namely $\mathrm{W}$ which is perfectly elastically isotropic and Au which is strongly elastically anisotropic, and for a nanometric $\mathrm{W} / \mathrm{Au}$ multilayers. The experimental measurements show that the model capture the elastic anisotropy rather well even for a nanometric multilayer stacking (period of $12 \mathrm{~nm}$ ) and that the elastic anisotropy of W/Au multilayer is more pronounced than the ones of the two components for a fraction of $50 \%$. This enhanced anisotropy is discussed in view of the multilayer microstructure.
\end{abstract}

\section{Introduction}

Crystals exhibit elastic anisotropy of varying degree. This local or intrinsic elastic anisotropy induces a macroscopic anisotropy in case of polycrystalline textured materials. These effects are very important for thin films or coatings. Indeed the deposition techniques, namely the physical vaport deposition (PVD), chemical vapour deposition (CVD) or electrodeposition, often lead to highly textured polycrystalline films [1]. These variations in crystallographic orientation associated to local crystalline elastic anisotropy have a pronounced effect on the overall mechanical response of thin films. Hence they have to be known and control because of their implications on the reliable operation of micro-electronic and micro-electromechanical systems. Due to the constraints of the applicabilities, thin films or coatings exhibit different structural configurations, i.e. mono-layers, multilayers with two or more components, and the sizes of structures decrease down to a few nanometers. Thus, in light of ongoing efforts to reduce characteristic feature sizes in such systems, it is imperative to study mechanical properties of materials at very small scale at very small scales [2-9]. At small dimensions, interfaces and singular structures are intrinsic to thin films, and mechanical modelling can be quite complex. In structural mechanics, the configurations of thin films or coatings are identified as plates or shells; but the composite structure and the specific behaviour of polycrystalline material composed of grain of a few nanometer should be also taken into account for describing mechanical behaviour of thin films and

\footnotetext{
* Corresponding author.

E-mail address: faurie@univ-paris13.fr (D. Faurie).
} 
multilayers. Materials are often assumed to behave uniformly, exhibiting equal strength in all directions, because most of them have a polycrystalline structure. However, the anisotropy of the individual crystals is smoothed out only in the presence of a large number of grains having a random distribution of orientations. Actually, a macroscopic anisotropy usually remains due to the existence of preferred orientations. Its magnitude depends upon the statistical distribution of grain orientations, i.e. the "crystallographic texture" more simply called the texture. This governs the extremes that a single-crystal of the material under consideration can exhibit during directional tests. Strong fiber textures are usually obtained in Physical Vapor Deposition (PVD) and have been shown to be macroscopically elastically anisotropic [10,11]. To describe the effective elastic behaviour of such polycrystalline films, it is convenient to apply elastic grain interaction models in order to describe stress and strain distribution in polycrystalline thin films. The elastic grain interaction models commonly used are Reuss [12], Voigt [13], Neerfeld-Hill [14,15], and Self-Consistent model [16,17]. The Reuss and Voigt models involve extreme grain-interaction assumptions: either the strain tensor (Voigt) or the stress tensor (Reuss) is assumed to be equal for all crystallites. Furthermore, Neerfeld and Hill found on an empirical basis that the arithmetic averages of elastic constants calculated according to the models of Voigt and Reuss are generally in good agreement with experimental data [18]. The Self-Consistent model is the most representative model of elastic-grain interaction in polycrystals, but is mathematically more complex. Moreover, considering a multilayer, the effective elastic constants of the whole material will depend on the properties and on the volume fractions of each kind of layers [7]. The resulting global symmetry of a multilayer composed of fiber-textured sub-layers is hexagonal-like with 5 independent effective stiffnesses. Usually, elastic anisotropy is analysed in terms of Zener anisotropy index [19] that applies only for cubic symmetry. The cases of lower symmetry (orthorhombic, tetragonal, hexagonal) are the subject of ongoing researches [20-24]. Recently, a general approach has been proposed [22] and applied successfully for a hexagonal ternary system [24]. The aim of the present paper is to study the elastic anisotropy in fiber-textured thin films (W and Au films, $\mathrm{W} / \mathrm{Au}$ multilayer film), whose effective symmetry is hexagonal. Especially, the effect of the multilayer stacking structure on global elastic anisotropy is investigated. The choice of tungsten and gold as model systems is motivated by the relative anisotropy of those cubic materials, i.e. perfect isotropy for $\mathrm{W}$ and strong anisotropy for Au and by the fact that those elements are immiscible at equilibrium. After reminding the multiscale modelling of effective elastic behaviour of such structures, a confrontation with acoustic measurements (Brillouin light scattering (BLS), picosecond ultrasonics (PU)) is made. The results show that a macroscopic two-scale transition model may be used to estimate the elastic anisotropy of nanometric multilayers and that the stacking design of a multilayer can induce a more pronounced anisotropy than the ones of each components.

\section{Effective elastic properties of a perfect polycrystalline multilayer}

This section reviews mechanical analysis of a perfect multilayer. The microstructure of the studied specimens can be described at different scales. At the larger scale, the specimen consists of different metallic polycrystalline layers that are formed themselves by nanometre sized grains (Fig. 1).

First, one has to evaluate the distribution of the applied strains and stresses in the metallic layers. This is a rather simple problem as long as each metallic layer can be replaced by a homogenous elastic layer of similar properties to the actual polycrystalline layer. Then, within each metallic layer, the strain/stress is concentrated heterogeneously in grains, or in particular regions within grains, depending on numerous factors including the local lattice orientation. The elastic behaviour can be written at each scale:

(i) grain scale:

$$
\boldsymbol{\sigma}=\mathbf{C}: \varepsilon
$$

(ii) layer scale:

$$
\overline{\boldsymbol{\sigma}}=\widetilde{\mathbf{C}}: \overline{\boldsymbol{\varepsilon}}
$$

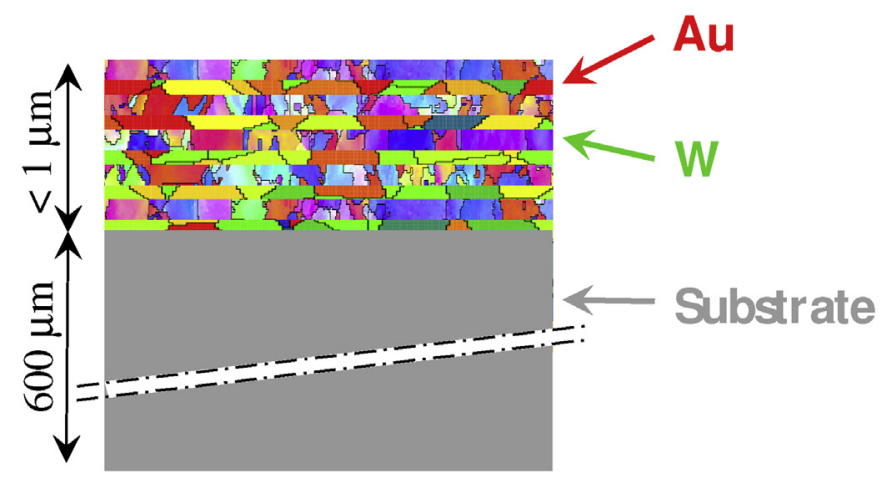

Fig. 1. Sketch of a multilayer composed of polycrystalline nanometric layers. 
(iii) multilayer scale:

$$
\overline{\overline{\boldsymbol{\sigma}}}=\widetilde{\widetilde{\mathbf{C}}}: \overline{\overline{\boldsymbol{\varepsilon}}}
$$

with $\mathbf{C}$ being the elastic stiffness tensor in the grains of the metallic layers; $\widetilde{\mathbf{C}}$ and $\widetilde{\widetilde{\mathbf{C}}}$ the effective stiffness tensors of each layer and of the specimen, respectively.

\subsection{Elastic behaviour of individual layers}

Polycrystalline metallic layers are made of a collection of grains exhibiting anisotropic properties and different crystallographic orientations. That is, these grains react differently to the applied stress $\bar{\sigma}_{i j}$. This gives rise to the building of complex mechanical interactions which have a large influence on the overall polycrystalline behaviour and on the associated distributions of stress and strain in the different grains. An important feature is the development of strain and stress heterogeneities inside the grains which are essential for the prediction of the effective behaviour [25]. The stress heterogeneity can be characterized by the stress localization tensor $\mathbf{B}$ defined as:

$$
\boldsymbol{\sigma}=\mathbf{B}: \overline{\boldsymbol{\sigma}}
$$

so that $\mathbf{B}$ can be interpreted as the ratio between local stress $\boldsymbol{\sigma}$ and average stress in each layer $\overline{\boldsymbol{\sigma}}$. The difficulty in any micromechanical approach is to find this localization tensor. Once it is known, local stress and strain can be determined, as well as the effective behaviour of the considered material. Obviously, B depends on the anisotropy of the local behaviour and on the microstructure of the specimen which may be complex.

The self-consistent (SC) model is often used to estimate the mechanical properties of materials and also to interpret diffraction data. As for all other mean-field homogenization methods, this model is based on a statistical description of the microstructure of the polycrystalline aggregates. Since the SC model provides the exact response of perfectly disordered microstructures, it is a good candidate for the modelling of polycrystalline materials, combining accuracy and relative simplicity. Complete expressions for the effective stiffness and localization tensors can be found in Brenner et al. [26] for general anisotropic situations. When only the volume fraction of the different grain orientations is known, i.e. no information concerning the geometric arrangement of these phases within the specimen is available then the tightest bounds that can be derived are the Reuss and the Voigt bounds. In this work, the SC model has been used for taking into account of the heterogeneities in the layers accurately.

\subsection{Effective behaviour of a multilayer}

At the multilayered film scale, due to the small thickness of each layer and small grain size compared to the lateral extent of layers, we consider the specimen as an infinite laminate material with homogeneous stiffness $C$ for each layer. Therefore, the effective stiffness $C$ of the whole specimen can be calculated exactly for general anisotropy of the layers [27]:

$$
\left[\sigma_{0}\left(\sigma_{0} \mathbf{I}-\widetilde{\widetilde{\mathbf{C}}}\right)^{-1}-\boldsymbol{\Gamma}\right]^{-1}=\left\langle\left[\sigma_{0}\left(\sigma_{0} \mathbf{I}-\widetilde{\mathbf{C}}\right)^{-1}-\boldsymbol{\Gamma}\right]\right\rangle
$$

with $\sigma_{0}$ an arbitrary scalar, I the identity tensor, and $\boldsymbol{\Gamma}$ a geometrical tensor depending only on the orientation of the specimen surface. 〈.) is the average over the whole specimen volume. The knowledge of $\widetilde{\mathbf{C}}$ enables the calculation of the average stresses $\overline{\boldsymbol{\sigma}}$ and strains $\overline{\boldsymbol{\varepsilon}}$ in each layer and are such that $\bar{\varepsilon}_{11}=\overline{\bar{\varepsilon}}_{11}, \bar{\varepsilon}_{22}=\overline{\bar{\varepsilon}}_{22}, \bar{\varepsilon}_{12}=\overline{\bar{\varepsilon}}_{12}, \bar{\sigma}_{i 3}=\overline{\bar{\sigma}}_{i 3}$ with $\overline{\bar{\sigma}}_{i j}$ and $\overline{\bar{\varepsilon}}_{i j}$ the average stresses and strains at the specimen scale. In this model, the interfaces between layers are assumed to be perfect, that is, infinitesimally thin and are not deformed by shear, and the displacements remain continuous through the interfaces.

\subsection{Numerical illustration}

Fig. 2 shows the calculations (dotted lines) of effective elastic constants for Au/W multilayers as a function of volume fraction of W; single-crystal elastic constants given in Table 1 are used in the model. Obviously, when W ratio (defined as W thickness divided by total thickness) is null, we calculate the elastic constants of a Au single-layer and when W ratio is equal to 1 , we calculate the elastic constants of a $\mathrm{W}$ single-layer with $\widetilde{\mathbf{C}}=\widetilde{\mathbf{C}}$. Reminding Au is locally elastically anisotropic (Zener anisotropy index $=2.8$ ), the Au layers are macroscopically anisotropic if textured. The actual strong $\{111\}$ fiber-texture of Au layers or film has been taken into account in the model.

This is why the values are different on the $c_{i j}$-axes of Fig. 2 even if gold is a cubic material. On the contrary, W layers or film can have any arbitrary texture; indeed body-centered cubic $\alpha$-W is known to be perfectly isotropic at the grain scale, so that the texture is not influent on the effective elastic properties at the layer scale $(\mathbf{C}=\widetilde{\mathbf{C}}=\widetilde{\widetilde{\mathbf{C}}})$. Because of the fiber texture, the W/Au multilayers are transversely isotropic for all the W ratios, with 5 independent effective elastic constants: $\widetilde{\widetilde{C}}_{11}, \widetilde{\widetilde{C}}_{12}, \widetilde{C}_{13}$, $\widetilde{\widetilde{C}}_{33}, \widetilde{\widetilde{C}}_{44}$ and $\widetilde{\widetilde{C}}_{66}=\frac{\left.\widetilde{\widetilde{C}}_{11}-\widetilde{\widetilde{C}}_{12}\right)}{2}$. When $\mathrm{W}$ ratio is equal to 1 , the sample is macroscopically isotropic, so that $\widetilde{\widetilde{C}}_{11}=\widetilde{\widetilde{C}}_{33}, \widetilde{\widetilde{C}}_{12}=\widetilde{\widetilde{C}}_{13}$ 

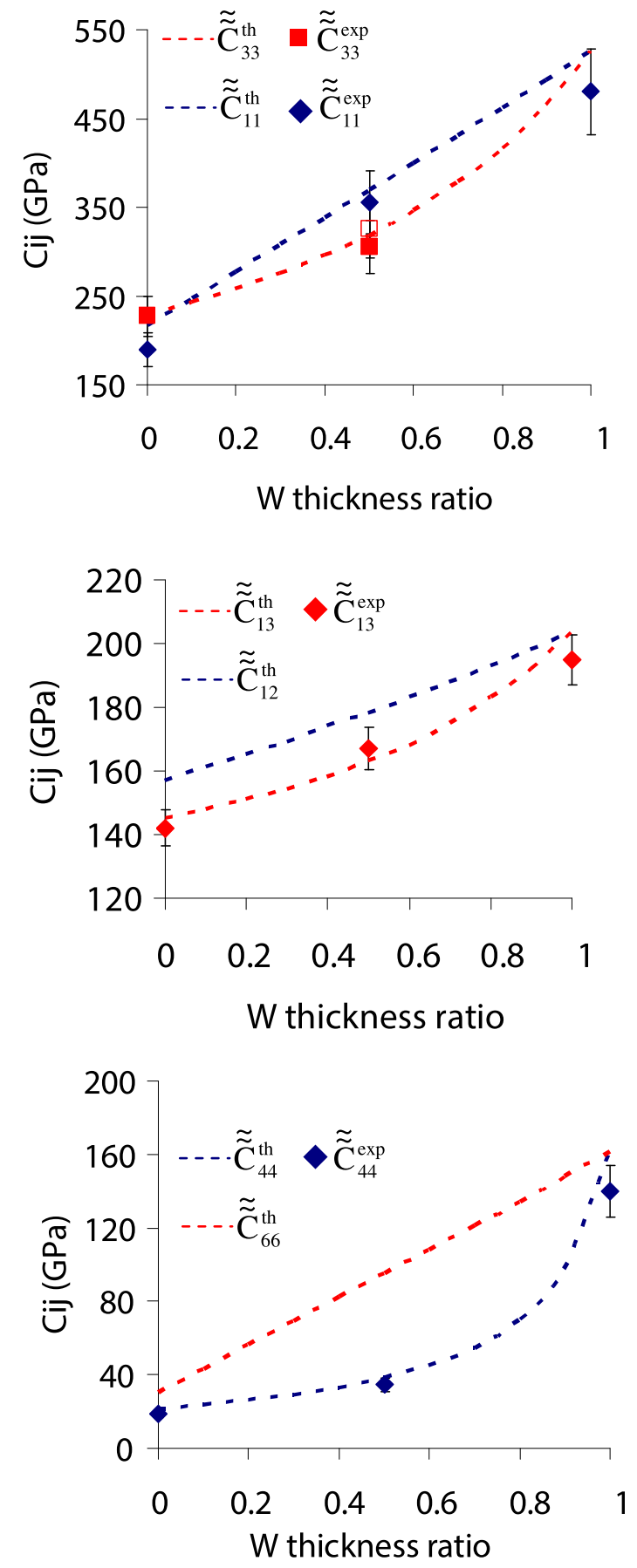

Fig. 2. Effective elastic constants as a function of $W$ thickness ratio in W/Au multilayers. W and Au films are defined for ratios equal to 1 and 0 respectively. (a) $\widetilde{\widetilde{C}}_{11}$ and $\widetilde{\widetilde{C}}_{33}$, (b) $\widetilde{\widetilde{\mathbf{C}}}_{12}$ and $\widetilde{\widetilde{\mathbf{C}}}_{13}$, (c) $\widetilde{\widetilde{\mathbf{C}}}_{44}$ and $\widetilde{\widetilde{\mathbf{C}}}_{66}$

Table 1

Gold and Tungsten single-crystals elastic constants [28].

\begin{tabular}{lcr}
\hline Single-crystal & Tungsten & Gold \\
\hline$C_{11}(\mathrm{GPa})$ & 525.4 & 192.3 \\
$C_{12}(\mathrm{GPa})$ & 204.3 & 162.9 \\
$C_{44}(\mathrm{GPa})$ & 160.5 & 42.0 \\
\hline Zener anisotropy index & 1.0 & 2.8 \\
\hline
\end{tabular}


and $\widetilde{\widetilde{C}}_{44}=\widetilde{\widetilde{C}}_{66}$. Since $\mathrm{W}$ is stiffer than Au, all the constants increase with the W ratio. The evolution is linear for the "in-plane" elastic constants $\left(\widetilde{\widetilde{C}}_{11}, \widetilde{\widetilde{C}}_{12}\right.$ and $\left.\widetilde{\widetilde{C}}_{66}\right)$ while it is quadratic for "out-of-plane" ones $\left(\widetilde{\widetilde{C}}_{13}, \widetilde{\widetilde{C}}_{33}\right.$ and $\left.\widetilde{\widetilde{C}}_{44}\right)$. It should be noted that the differences between $\widetilde{\widetilde{C}}_{11}$ and $\widetilde{\widetilde{C}}_{33}, \widetilde{\widetilde{C}}_{12}$ and $\widetilde{\widetilde{C}}_{13}, \widetilde{\widetilde{C}}_{44}$ and $\widetilde{\widetilde{C}}_{66}$, are characteristic of elastic anisotropy at the macroscopic scale. Especially, contrary to one might expect at a first sight, the multilayer $\mathrm{W} / \mathrm{Au}(\mathrm{W}$ ratio $=0.5)$ shows a more pronounced elastic anisotropy than the Au single-layer while W single-layer is intrinsically elastically isotropic. In order to confirm this,
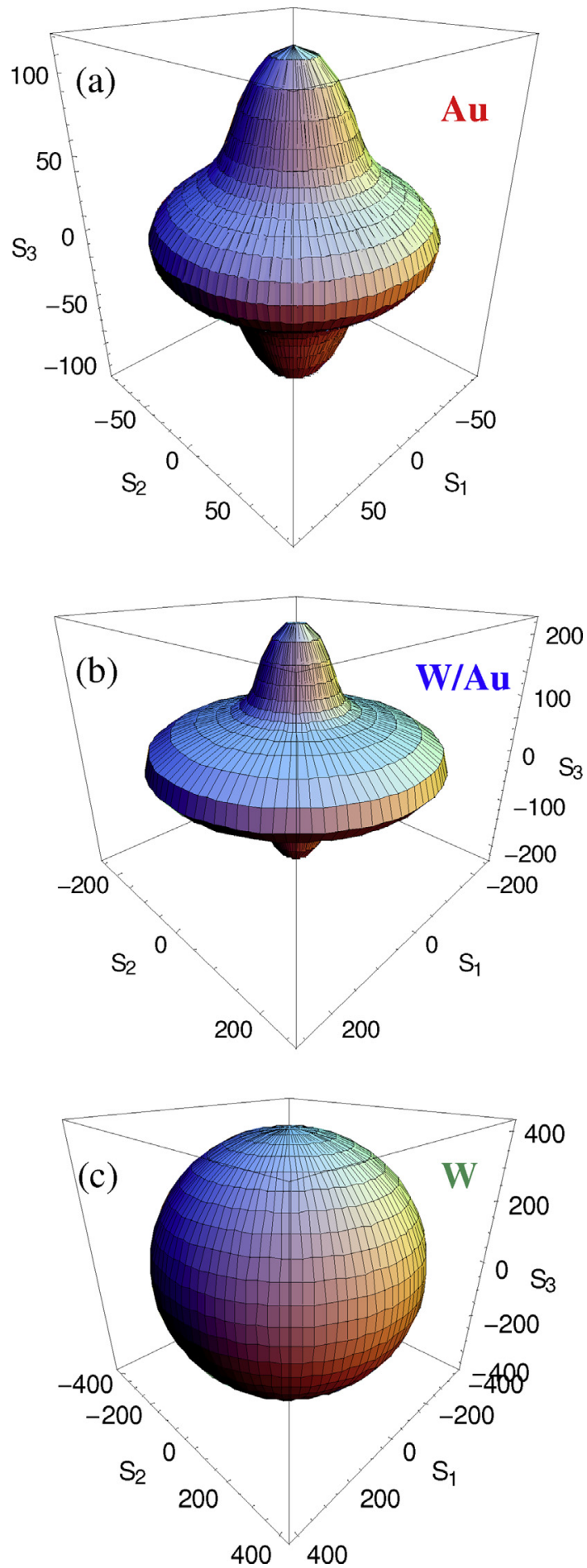

Fig. 3. Three-dimensional surface parametric plot of Young's modulus for (a) \{111\}-fiber-textured Au film, (b) W/Au multilayer, (c) W film. 
we have calculated the direction dependent Young's modulus (Fig. 3$)$ in the sample reference frame $\left(S_{1}, S_{2}, S_{3}\right)$, as described in Faurie et al. [18], for three $\mathrm{W}$ thickness ratios $(0,0.5$ and 1$)$. The sphericity of 3D parametric surfaces is characteristic of macroscopic elastic isotropy, as found for the W single layer (Fig. 3-c). Moreover, we find that Young's modulus of W/Au multilayer (Fig. 3-b) is indeed more direction-dependent than the Au single-layer (Fig. 3-a), as foreseen in Fig. 2. Fig. 4 shows the comparison of the 3 parametric surfaces in the $\left(S_{1}, S_{3}\right)$ plane. It is clearly shown that the stiffness of the film increases with the $\mathrm{W}$ thickness ratio. Moreover the macroscopic anisotropy already present in the pure Au single-layer is not smoothed by the presence of $\mathrm{W}$ in a W/Au multilayer stacking, and it reaches a maximum for thickness ratios around 0.5. This is due to the anisotropic character of the microstructure, i.e. the multilayer stacking design.

\section{Experimental details}

\subsection{Thin film deposition and microstructure}

$200 \mathrm{~nm}$ thick W and $700 \mathrm{~nm}$ thick Au single-layers and a W/Au multilayer have been magnetron sputter deposited on Si substrate. The last one was composed of 58 periods (alternatively deposited W sub-layers $(6 \mathrm{~nm})$ and Au sub-layers $(6 \mathrm{~nm})$ ), so that the total thickness was about $700 \mathrm{~nm}$. The thickness of sublayers has been chosen to limit interfacial effects, already observed for smaller periods in similar systems $[29,30]$ and to have a polycrystalline materials with a truly nanometric grain size (smaller than $6 \mathrm{~nm}$ ) well below 20-30 nm where grain boundaries activities become dominant, as suggested by numerous molecular dynamics simulations [31-33]. Even elastic constants may change in the nanometric range [34-36]. Noticeably the nanomaterials synthesized by energetic physical vapour deposition process does not contain the different type of defects structure such as the nanocrystalline metals prepared by pulsed electrodeposition or inert gas condensation, e.g. nanovoids, twins, impurities [37-39]. Using x-ray diffraction technique, the Au layers were found to be $\{111\}$-fiber textured while the $\mathrm{W}$ layers were found to be $\{110\}$-fiber textured.

\subsection{The Brillouin light scattering technique}

Measurement of the whole set of constants is usually out of the reach of conventional static techniques, which can give information about directional elastic modulus. On the other hand, the use of acoustic techniques based on surface acoustic waves (SAW) presents technical difficulties connected with the fabrication of the acoustical delay line. In addition, one has to consider that in the frequency range commonly used, the large values of the acoustic wavelength, compared to the film thickness, impose a careful consideration of the effect of the substrate on the propagation of the SAW [40]. During the last twenty years, BLS has proved to be very efficient for achieving a complete elastic characterization of thin films and multilayered structures [41-43]. In a BLS experiment, a monochromatic light beam is used to probe and to reveal acoustic phonons which are naturally present in the medium under investigation. The power spectrum of these phonons is mapped out from frequency analysis of the light scattered within a solid angle. Because of the wave-vector conservation in the phonon-photon interaction, the wavelength of the revealed elastic waves is of the same order of magnitude as that of light. This means that the wavelength is much larger than the interatomic distances, so that the material can be described as a continuum within an effective-medium approach. The BLS spectra were taken in air at room temperature, with typical acquisition times of 1-2 h.

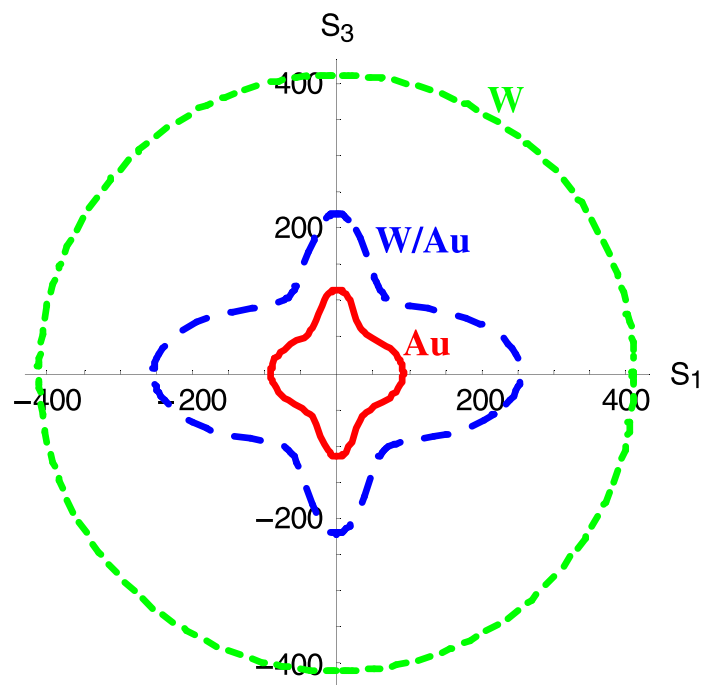

Fig. 4. Two-dimensional surface parametric plot of Young's modulus (in GPa) in a transverse plane of the samples $\left(\mathbf{S}_{\mathbf{1}}, \mathbf{S}_{\mathbf{3}}\right)$. 
The light source was an $\mathrm{Ar}+$ laser on a single mode of the $514.5 \mathrm{~nm}$ line. $300 \mathrm{~mW}$ of p-polarized light was focused on the surface of the sample and the scattered light was analysed by means of a Sandercok-type $3+3$ pass tandem Fabry-Perot interferometer. Here, we used the backscattering interaction geometry, so that the value of the wave vector of the probed surface acoustic waves is fixed experimentally to the value $Q_{/ /}=2 \mathrm{k} \sin (\vartheta)$, where $\mathrm{k}$ denotes the optical wave vector in air and $\vartheta$ the incidence angle of the light beam.

\section{Results and discussion}

For nearly opaque films with thicknesses lower or similar to the acoustic wavelength (300-400 nm), supported by substrates with acoustic phase velocities higher than that of the films (slow film/fast substrate), such as a metallic film on a Silicon substrate, we can only observe (see Fig. 5) the surface acoustic waves with a sagittal polarization: the Rayleigh wave (R) and the so-called Sesawa guided waves $\left(S_{1}\right.$ to $\left.S_{n}\right)$ the corresponding phase velocities being measured. In the present case, four of the five effective elastic constants, namely $\widetilde{\widetilde{C}}_{11}, \widetilde{\widetilde{C}}_{13}, \widetilde{\widetilde{C}}_{33}$, and $\widetilde{\widetilde{C}}_{44}$, influence the Rayleigh and Sezawa modes, so that
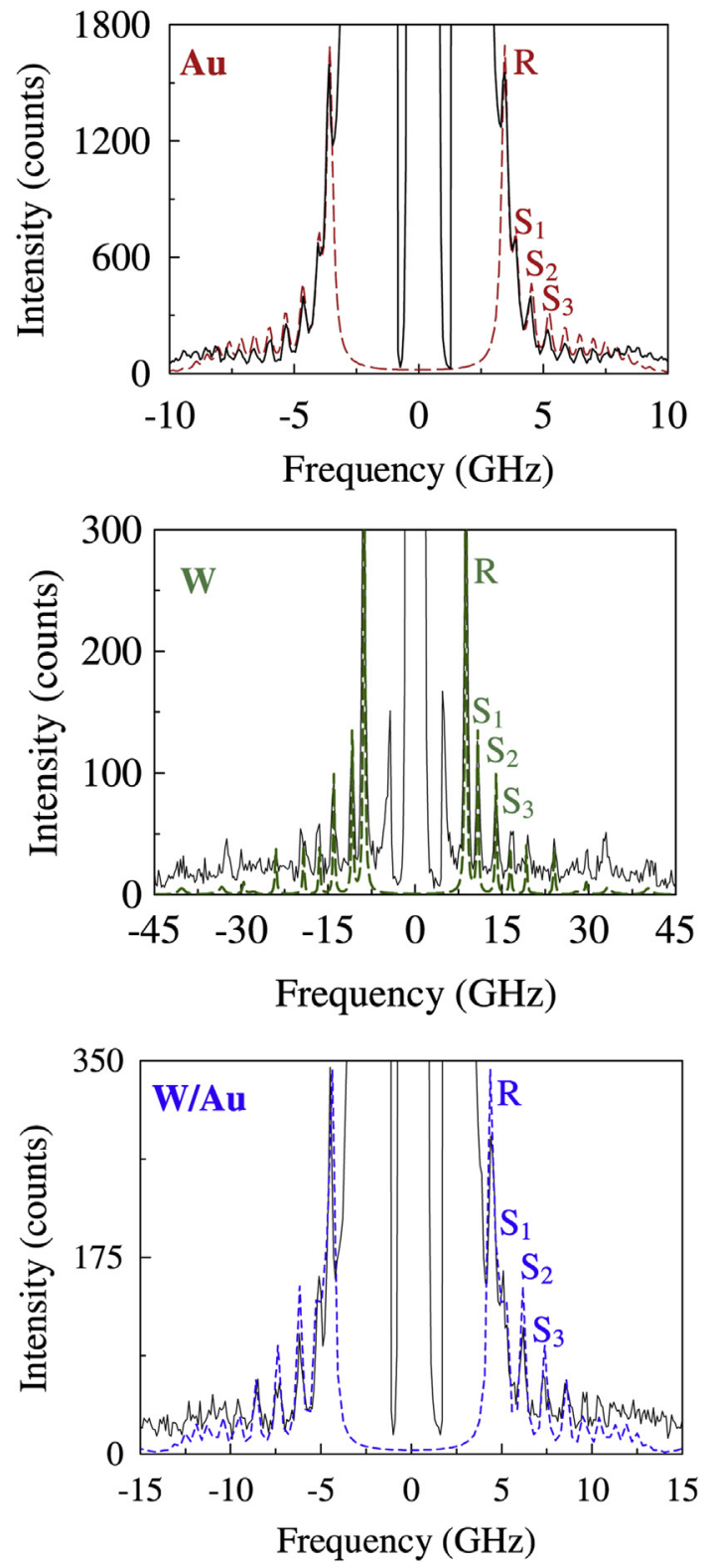

Fig. 5. Experimental and numerical Brillouin Light Scattering spectra for (a) Au film, (b) W film and (c) W/Au multilayer film. Effective elastic constants $\widetilde{\widetilde{C}}_{11}, \widetilde{\widetilde{C}}_{13}$, $\widetilde{\widetilde{C}}_{33}$, and $\widetilde{\widetilde{C}}_{44}$ are estimated from the fitting of experimental. 
Table 2

Experimental and theoritical effective elastic stiffnesses, shear and universal anisotropic indexes, for the three studied films showing different tungsten ratio (i.e. different thicknesses). The maximum error on elastic constants determination is $10 \%$.

\begin{tabular}{|c|c|c|c|}
\hline W Ratio & 0 & 0.5 & 1 \\
\hline$\widetilde{\widetilde{C}}_{11}^{\text {th }}(\mathrm{GPa})$ & 217 & 369 & 525 \\
\hline$\widetilde{\widetilde{C}}_{11}^{\exp }(\mathrm{GPa})$ & 190 & 355 & 480 \\
\hline$\widetilde{\widetilde{C}}_{12}^{\exp }(\mathrm{GPa})$ & 157 & 178 & 204 \\
\hline$\widetilde{\widetilde{C}}_{13}^{\text {th }}(\mathrm{GPa})$ & 145 & 163 & 204 \\
\hline$\widetilde{\widetilde{C}}_{13}^{\exp }(\mathrm{GPa})$ & 142 & 167 & 195 \\
\hline$\widetilde{\widetilde{C}}_{33}^{\text {th }}(\mathrm{GPa})$ & 228 & 318 & 525 \\
\hline$\widetilde{\widetilde{C}}_{33}^{\exp }(\mathrm{GPa})$ & 227 & $305 / 325^{a}$ & 480 \\
\hline$\widetilde{\widetilde{C}}_{44}^{\text {th }}(\mathrm{GPa})$ & 22 & 38 & 161 \\
\hline$\widetilde{\widetilde{C}}_{44}$ (GPa) & 19 & 35 & 140 \\
\hline$\widetilde{\widetilde{C}}_{66}^{\text {th }}(\mathrm{GPa})$ & 30 & 95 & 161 \\
\hline$A^{\text {shear,th }}$ & 0.55 & 0.42 & 1.00 \\
\hline$A^{\text {shear }, \exp }$ & 0.56 & 0.42 & 0.98 \\
\hline$A^{U, t h}$ & 0.52 & 1.14 & 0 \\
\hline
\end{tabular}

a Obtained by picosecond ultrasonic.

they can be evaluated by a best-fit procedure of the experimental velocities to the calculated dispersion curves. The fifth elastic constant could be determined from measurements of the phase velocity of shear horizontal modes (Love modes) but are not observed here because of their low scattering efficiency. Fig. 5 shows the BLS spectra for the three samples. The continuous and dotted lines show the experimental and simulated spectra respectively. Actually, since W and Au have very similar mass density $\left(19.3 \mathrm{~g} / \mathrm{cm}^{3}\right)$, the difference of surface mode frequencies between the three graphs depends only on the difference in elastic stiffnesses and in the whole thickness of the films. In this study, the singularity of W single-layer spectrum is mainly due to its small thickness as compared to Au single-layer and W/Au multi-layer ones. For all spectra, the adjustment of surface modes allows estimating the 4 elastic constants cited above.

The results are reported in Fig. 2 (square and losange symbols) and Table 2, and are compared to the calculations (dotted lines) already described in section 2.

The agreement between experimental and calculated stiffnesses is good, since the evolution of stiffnesses as a function of $\mathrm{W}$ thickness ratio is well described. This observation confirms the specific macroscopic elastic anisotropy induced by the multilayer microstructure, which induces a non-linear evolution of "out-of-plane" elastic stiffnesses, such as $\widetilde{C}_{13}, \widetilde{C}_{33}$, and $\widetilde{C}_{44}$. Because of the strong fiber-texture, the elastic anisotropy has to be quantified here for an hexagonal symmetry, i.e. in terms of shear anisotropic index as reviewed recently by Ivanovskii [23] and is given by:

$$
A^{\text {shear }}=\frac{4 \widetilde{\widetilde{C}}_{44}}{\widetilde{\widetilde{C}}_{11}+\widetilde{\widetilde{C}}_{33}-2 \widetilde{\widetilde{C}}_{13}}
$$

The perfect isotropy is defined at $A^{\text {shear }}=1$ as it is the case for the Zener anisotropy index defined for cubic materials. The experimental and theoretical values for the three samples are shown in Fig. 6 and Table 2.

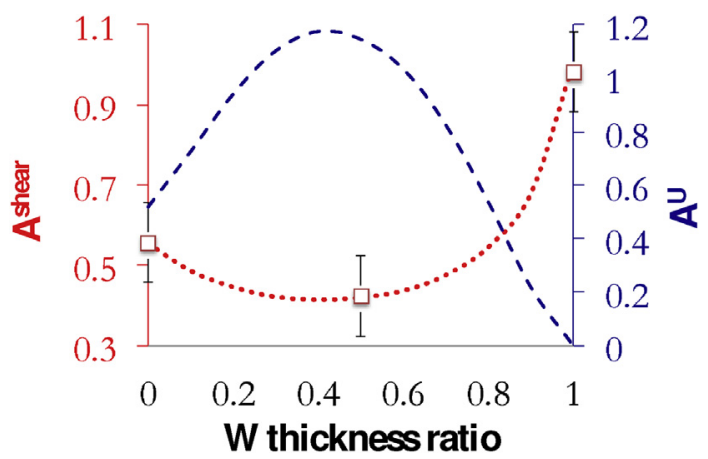

Fig. 6. Anisotropy indexes as a function of tungsten ratio. Isotropy is defined for $\mathbf{A}^{\text {shear }}=\mathbf{1}$ and $\mathbf{A}^{\mathbf{U}}=0$. 
The theoretical curve passes through a minimum for a $\mathrm{W}$ thickness ratio of approximately 0.4 , which corresponds to the highest elastic anisotropy, close to the one of the studied multilayer. The agreement between modelling and experiments is good, for extrema W ratios, i.e. pure W film and pure Au film. Furthermore, one can consider the universal anisotropy index which is defined by Ref. [22]:

$$
A^{U}=\widetilde{\widetilde{\mathbf{C}^{\mathbf{V}}}}:\left(\widetilde{\widetilde{\mathbf{C}^{\mathbf{R}}}}\right)^{-1}-6
$$

with $\widetilde{\mathbf{C}^{\mathbf{V}}}$ the stiffness tensor calculated using Voigt model [13] and $\widetilde{\mathbf{C}^{\mathbf{R}}}$ the stiffness tensor calculated using Reuss model [12]. As the calculation of $A^{U}$ requires the knowledge of all elastic stiffnesses, it can only be calculated theoretically in the present case. This index cannot be directly compared to the shear anisotropy index (for which perfect isotropy is defined at $A^{U}=0$ ), but the maximum anisotropy is found at the same thickness ratio of the multilayer (about 0.4). Such enhanced anisotropy is expected to appear in laminated structures, which amplitude is determined by the mechanical contrast between phases, and not by the anisotropy of each one.

\section{Conclusion}

We have studied both numerically and experimentally the elastic constants of Au and W single-layer and W/Au multilayer. The stiffnesses' evolution predicted by the two-scale transition model has been confirmed experimentally by Brillouin light scattering for the three films. This macroscopic stiffness evolution is either linear or non-linear with the $\mathrm{W}$ thickness ratio in W/Au multilayers. Based on the stiffness values, the anisotropy of the three films has been investigated. The multilayer exhibits the more pronounced anisotropy for a $\mathrm{W}$ fraction of $40 \%$. This result is related to the macroscopic elastic anisotropy induced by the specific arrangement of sublayers and by the induced elastic field heterogeneities. Especially, it is noteworthy that a W/Au multilayer is found to be more elastically anisotropic than Au singlelayer, even though W is perfectly isotropic. This enhanced anisotropy is expected to be observed in multilaminates with high mechanical constrast between constituents. Moreover, even if the mechanical response of nanometric multilayers is likely to be more influenced by interatomic potentiels and interface energy than by macroscopic mechanical properties, the present results show that a macroscopic two-scale transition model may be utilized to optimise the mechanical anisotropy of truly nanometric multilayers by tuning the component fractions.

\section{References}

[1] I. Petrov, P.B. Barna, L. Hultman, J.E. Greene, J. Vac. Sci. Technol. A 21 (2003) S117.

[2] H. Espinosa, B. Prorok, M. Fischer, J. Mech. Phys. Solids 51 (2003) 47.

[3] M. Haque, M. Saif, Acta Mater. 51 (2003) 3053.

[4] H. Huang, F. Spaepen, Acta Mater. 48 (2000) 3261.

[5] W. Xu, J.S. Yang, T.J. Lu, Mater. Des. 32 (2011) 154.

[6] E. Arzt, G. Dehm, P. Gumbsch, O. Kraft, D. Weiss, Prog. Mater. Sci. 46 (2001) 283.

[7] C. Rossignol, B. Perrin, B. Bonello, P. Djemia, P. Moch, H. Hurdequint, Phys. Rev. B 70 (094102) (2004).

[8] J. Keckes, M. Bartosik, R. Daniel, C. Mitterer, G. Maier, W. Ecker, J. Vila-Comamala, C. David, S. Schoeder, M. Burghammer, Scr. Mater. 67 (2012) 748.

[9] K. Tu, Phys. Rev. B 45 (1992) 1409.

[10] D. Faurie, P.-O. Renault, E.L. Bourhis, P. Goudeau, Acta Mater. 54 (2006) 4503.

[11] S. Matthies, H. Priesmeyer, M. Daymond, J. Appl. Crystallogr. 34 (2001) 585.

[12] A. Reuss, Z. Angew, Math. Mech. 9 (49) (1949).

[13] W. Voigt, Lehrbuch der Kristallphysik, Verlag, Leipzig, Teubner, 1910.

[14] H. Neerfeld, Mitt. K. Wilh. Inst. Eisenforschg 24 (61) (1942).

[15] R. Hill, Proc. Phys. Soc. Lond. 6534965 (349) (1952).

[16] E. Krï œner, Z Phys. 151 (504) (1958).

[17] J.D. Eshelby, Proc. R. Soc. A 241 (376) (1957).

[18] D. Faurie, P. Djemia, E.L. Bourhis, P.-O. Renault, Y. Roussigne, S. Cherif, R. Brenner, O. Castelnau, G. Patriarche, P. Goudeau, Acta Mater. 58 (4998) (2010).

[19] C. Zener, Elasticity and Anelasticity of Metals, University of Chicago, Chicago, 1948.

[20] H. Ledbetter, A. Migliori, J. Appl. Phys. 100 (063516) (2006).

[21] K. Lau, A.K. McCurdy, Phys. Rev. B 58 (8980) (1998).

[22] S.I. Ranganathan, M. Ostoja-Starzewski, Phys. Rev. Lett. 101 (055504) (2008).

[23] A. Ivanovskii, Prog. Mater. Sci. 57 (184) (2012).

[24] D. Groh, W.J. Slough, R. Pandey, S.P. Karna, D. Dandekar, Phys. Rev. B 83 (115122) (2011).

[25] R. Brenner, R. Lebensohn, O. Castelnau, Intern. J. Solids Struct 46 (3018) (2009).

[26] R. Brenner, O. Castelnau, L. Badea, Proc. R. Soc. A 460 (3589) (2004).

[27] G.W. Milton, The Theory of Composites, Cambridge University Press, 2001.

[28] J.C. Smithells, Metals Reference Book, fifth ed., Butterworths, London, 1976.

[29] P. Goudeau, P. Villain, T. Girardeau, P.-O. Renault, K.-F. Badawi, Scr. Mater. 50 (723) (2004).

[30] M. Jaouen, J. Pacaud, C. Jaouen, Phys. Rev. B 64 (144106) (2001).

[31] J. Schiï ¿œtz, T. Vegge, F.D. Di Tolla, K.W. Jacobsen, Phys. Rev. B 60 (11971) (1999).

[32] F. Sansoz, K.D. Stevenson, Phys. Rev. B 83 (224101) (2011).

[33] H. Van Swygenhoven, P.M. Derlet, Phys. Rev. B 64 (224105) (2001).

[34] R. Dingreville, J.M. Qu, M. Cherkhaoui, J. Mech. Phys. Solids 53 (1827) (2005). 
[35] P. Villain, P. Goudeau, P.-O. Renault, K.F. Badawi, Appl. Phys. Lett. 81 (4365) (2002).

[36] H. Ogi, M. Fujii, N. Nakamura, T. Yasui, M. Hirao, Phys. Rev. Lett. 98 (195503) (2007).

[37] K. Lu, L. Lu, S. Suresh, Science 324 (349) (2009).

[38] S.V. Petegem, F.D. Torre, D. Segers, H.V. Swygenhoven, Scr. Mater. 48 (17) (2003).

[39] Y.M. Wang, R.T. Ott, T. van Buuren, T.M. Willey, M.M. Biener, A.V. Hamza, Phys. Rev. B 85 (014101) (2012).

[40] H. Coufal, K. Meyer, R. Grygier, M. Vries, D. Jenrich, P. Hess, Appl. Phys. A 59 (1994).

[41] P. Djemia, M. Benhamida, K. Bouamama, L. Belliard, D. Faurie, G. Abadias, Surf. Coat. Tech. 215 (199) (2013).

[42] T. Pham, D. Faurie, P. Djemia, L. Belliard, E. Le Bourhis, P. Goudeau, F. Paumier, Appl. Phys. Lett. 103 (041601) (2013).

[43] G. Abadias, Ph Djemia, L. Belliard, Surf. Coat. Tech. 257 (129) (2014). 